\title{
Genomic Organization and Evolutionary Insights on GRP and NCR Genes, Two Large Nodule-Specific Gene Families in Medicago truncatula
}

\author{
Benoit Alunni, Zoltan Kevei, Miguel Redondo-Nieto, Adam Kondorosi, Peter Mergaert, and \\ Eva Kondorosi
}

Institut des Sciences du Végétal, Centre National de la Recherche Scientifique UPR2355, Bat 23, Avenue de la Terrasse, 91198 Gif-sur-Yvette cedex, France

Submitted 13 March 2007. Accepted 20 April 2007.

\begin{abstract}
Deciphering the mechanisms leading to symbiotic nitrogenfixing root nodule organogenesis in legumes resulted in the identification of numerous nodule-specific genes and gene families. Among them, NCR and GRP genes encode short secreted peptides with potential antimicrobial activity. These genes appear to form large multigenic families in Medicago truncatula and other closely related legume species, whereas no similar genes were found in databases of Lotus japonicus and Glycine max. We analyzed the genomic organization of these genes as well as their evolutionary dynamics in the $M$. truncatula genome. A total of 108 NCR and 23 GRP genes have been mapped that were often clustered in the genome. These included 29 new $N C R$ and 17 new GRP genes. Reverse transcription-polymerase chain reaction analyses of the novel genes confirmed their exclusive nodule-specific expression similar to the previously identified members. Protein alignments and phylogenetic analyses revealed traces of several duplication events in the history of $G R P$ and NCR genes. Moreover, microsyntenic evidences between $M$. truncatula and $L$. japonicus validated the hypothesis that these genes are specific for the inverted repeat-lacking clade of hologalegoid legumes, which allowed dating the appearance of these two gene families during the evolution of legume plants.
\end{abstract}

Nitrogen access in natural ecosystems is one of the key limiting factors for plant growth and development. To face and overcome this problem, most legume plants developed the ability to form symbiotic nitrogen-fixing nodules in association with rhizobia. The process of nodule development requires several recognition steps by both the bacteria and the plant. Nodule organogenesis is triggered by Rhizobium Nod factors in the host plant. Therefore, during recent years, surveys focused on the Nod factor signaling pathway, leading to the discovery of crucial genes playing roles in Nod factor recognition and signal transduction in the two model legumes Medicago trun-

\section{B. Alunni and Z. Kevei have contributed equally to this work.}

Corresponding author: E. Kondorosi; E-mail: Eva.Kondorosi@isv.cnrsgif.fr; Fax: +33(0)169823695.

Current address of M. Redondo-Nieto: Dpto. Biología. Facultad de Ciencias, Univ. Autónoma de Madrid, E-28049 Madrid, Spain.

All sequences corresponding to NCR and GRP family members mapped on the genome (mRNA and protein sequences) are submitted to GenBank and and are available under accession numbers EF414284 to EF414410. catula and Lotus japonicus (Stacey et al. 2006). Moreover, several hundreds of genes have been identified during the last decade, which were activated at different stages of symbiotic nodule development (El Yayahoui et al. 2004; Fedorova et al. 2002; Györgyey et al. 2000; Kouchi et al. 2004). Among them, two nodule-specific gene families encode secreted peptides. These are glycine-rich proteins (GRP; Kevei et al. 2002) and the cysteine-rich peptides (NCR; Mergaert et al. 2003). Beside their exclusive nodule-specific expression, GRP and NCR genes have also been shown to display a wide range of spatio-temporal patterns in the infected cells throughout nodule organogenesis.

GRP are known to be involved in many biological processes, including biotic and abiotic interactions between plants and their environment (Sachetto-Martins et al. 2000). Usually, the glycine content of GRP is $80 \%$ and glycine residues are ordered in typical motifs. Nevertheless, nodule-specific GRP are different; in general, they are shorter and contain fewer glycines (20 to $30 \%$ ), with no clear motif structures. The studied nodGRP in $M$. sativa and $M$. truncatula formed three groups based on the divergent glycine-rich regions. Such GRP were found in Vicia faba, Trifolium repens, and Medicago species (Kevei et al. 2002), all belonging to the inverted repeat-lacking clade (IRLC) of legumes. This clade of legume species was previously often referred to as galegoids or hologalegoids, but recent phylogenetic studies supported a more accurate definition of the clade as IRLC (Wojciechowski et al. 2004).

$N C R$ genes form an exceptionally large family consisting of more than 300 genes in M. truncatula (Fedorova et al. 2002; Mergaert et al. 2003). NCR genes code for short secreted peptides with four or six cysteine residues in conserved positions along the 50 to 60 amino acids of the mature peptide. They are similar to scorpion toxins, Conus spp. toxins, plant and animal defensins, or determinants of self incompability (Mergaert et al. 2003), which also form multigenic families and mostly play roles in interactions between different organisms, in pathogenicity, or innate immunity processes (Klotman and Chang 2006; Olivera 2006; Rodriguez de la Vega and Possani 2005; Silverstein et al. 2005) but can be involved in regulation of reproduction as well (Schopfer et al 1999; Watanabe et al 2000). NCR are members of a large cysteine cluster protein (CCP) family (Graham et al. 2004). This family of cysteinerich proteins comprises 10 subgroups, including the nodulespecific CCP (corresponding to NCR), the seed-specific CCP, and numerous ubiquitous CCP (harboring 4, 6, 8, or 10 conserved cysteines) (Graham et al. 2004; Silverstein et al. 2006). While NCR genes were found so far only in expressed sequence tag (EST) databases and genomic libraries of IRLC legumes 
(Mergaert et al. 2003), other types of $C C P$ were not specific for IRLC (e.g., Glycine max) and were identified also in nonlegume plants, such as Arabidopsis thaliana (Graham et al. 2004; Silverstein et al. 2006). The large number of NCR genes and the significantly divergent amino-acid sequences within the family are reminiscent of other gene families, such as disease resistance $(R)$ genes, which are also involved in interactions between organisms (Meyers et al. 2003).

The advanced sequencing projects of the two model legumes $M$. truncatula and L. japonicus allow comparative genomic studies. Though neither of them is entirely sequenced, a 54\% general syntenic relation between the two plants has been shown recently (Cannon et al. 2006), and detailed microsyntenic analysis can give new insights in the evolution of larger gene families.

The aim of our study was to describe gene structure and organization of GRP and NCR families in the M. truncatula genome. Therefore, genomic databases were screened to identify formerly studied and new members of these gene families. Using this strategy, we mapped 23 GRP and 108 NCR genes, including, respectively, 17 and 29 novel genes and confirmed their nodule-specific expression. Many of the GRP and NCR genes were found in clusters as the consequence of numerous local duplication events. These data were supported by their phylogenetic analysis and also by the evolution study of their signal peptide and mature domain. Moreover, evidences for microsyntenic relations between $M$. truncatula and $L$. japonicus were found in the neighboring genomic regions of $N C R$ and GRP genes, but the NCR and GRP clusters themselves were absent in the Lotus genome.

\section{RESULTS}

Nodule-specific GRP genes in M. truncatula.

The different protein sequences deduced from cDNAs of the MtnodGRP gene family (MtnodGRP1A, MtnodGRP1B, MtnodGRP1C, MtnodGRP2A, MtnodGRP2B, MtnodGRP3) (Kevei et al. 2002) were used for tBLASTn homology searches in the National Center for Biotechnology Information (NCBI) database. The searches resulted in three entirely sequenced $M$. truncatula genomic regions containing these genes. As the signal peptides were highly or well conserved in the three groups of GRP, these were used for tBLASTn searches to identify further potential GRP genes in the M. truncatula genome. This nodGRP gene hunting led to the discovery of additional members that were localized in six different genomic regions in total.

The previously described MtnodGRP1A, MtnodGRP1B, and MtnodGRPIC genes were on the same DNA contig, which contained, in addition, nine new MtnodGRPl genes
(MtnodGRP1D through MtnodGRPIL) (Fig. 1). On the other hand, the MtnodGRPIB mRNA turned out to be an alternatively spliced form of the MtnodGRPIA gene. The 11 MtnodGRPI genes were scattered in a $270-\mathrm{kb}$ region, but eight of them were within a 70-kb distance. Six of the 11 MtnodGRPl genes possessed EST sequences in the database but not the MtnodGRP1D, MtnodGRP1E, MtnodGRP1F, MtnodGRPIG, or MtnodGRPII genes.

The MtnodGRP2 contig on chromosome 2 clustered four genes (MtnodGRP2A to MtnodGRP2D) that were in the same orientation (Table 1). MtnodGRP2C and MtnodGRP2D were novel genes, but EST were found only for MtnodGRP2D. The MtnodGRP3 search revealed three homologous genes in the same region (MtnodGRP3A, MtnodGRP3B, MtnodGRP3C) and EST for MtnodGRP3A and MtnodGRP3B. The remaining $G R P$-related genes formed three new clusters located in three different genomic regions (Table 1); chromosome 2 carried two gene copies both for MtnodGRP5 (MtnodGRP5A and MtnodGRP5B) and for MtnodGRP6 (MtnodGRP6A and $M t n o d G R P B)$, whereas the unique MtnodGRP4 gene was on a region that has not yet been assigned to any chromosome of $M$. truncatula (designated as chromosome 0). EST were available for MtnodGRP4 and MtnodGRP5A.

\section{New NCR genes in the M. truncatula genome.}

In the EST databases, sequences were available for $311 \mathrm{NCR}$ genes (NCR001 to NCR00311) (Mergaert et al. 2003). In order to localize these NCR genes in the genome, BLASTn homology searches were performed on $M$. truncatula bacterial artificial chromosome (BAC) sequences. A total of 51 different genomic regions carrying 79 of the known $N C R$ genes were identified, and thus, the contigs often combined multiple $N C R$ genes (Fig. 1; Table 1).

To verify the existence of further NCR on the NCR-containing BAC clones, tBLASTn searches were performed. The typical cysteine motifs and signal peptide parts helped to find neighboring NCR genes (Graham et al 2004; Mergaert et al 2003). These searches resulted in the identification of 29 new $N C R$ genes (NCR312 to NCR340) that were present in the vicinity of known NCR genes on 17 different contigs (Table 1). Of the 29 NCR, 19 peptides harbored the four-cysteine and nine the six-cysteine motif. Of the new NCR genes, 26 had no EST. There were EST for the NCR314, NCR319, and NCR337 genes, which were, however, not annotated before as $N C R$. NCR323, NCR324, and NCR335 corresponded to the formerly described $C C P 1, C C P 3$, and $C C P 4$, respectively (Graham et al 2004). In the whole genome, 27 NCR contigs contained one, nine contained two, six contained three, three contained four,

\section{MtnodGRP1-contig (mth2-174i23, mth2-24k1, mth2-170k9) - chr5}

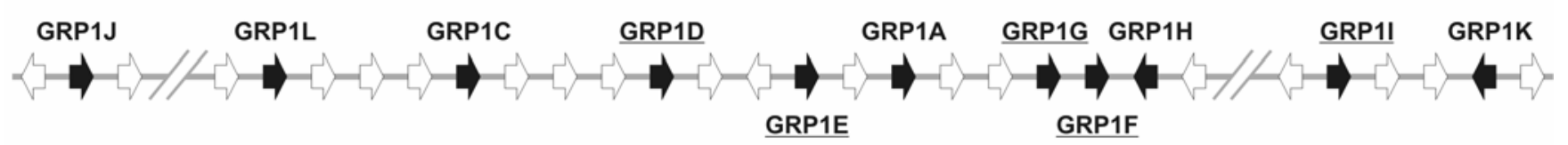

\section{NCR024/026/088/138/316/334-contig (mth2-12a23) - chr2}
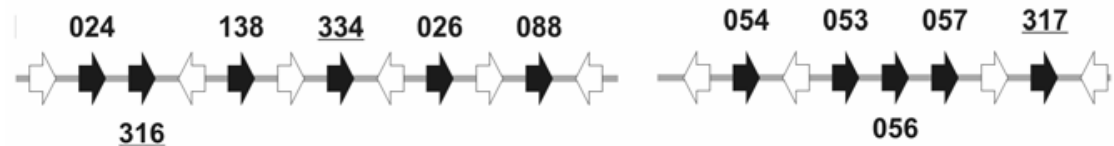

NCR239/325/326/327/328-contig
(mth2-192a14) - chr7

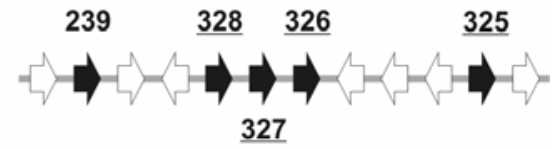

Fig. 1. Genomic organization of the MtnodGRP1 contig and three NCR contigs in Medicago truncatula. The Mtnod GRP1 contig contains 11 GRP1 genes (called GRP1A to GRP1L). The BAC clone numbers corresponding to the contigs are indicated between brackets and followed by the chromosome number. The location and orientation of genes are indicated by arrows. Genes and intergenic regions are not drawn at scale. Black arrows represent the GRP or NCR genes and their underlining indicates the absence of expressed sequence tags. Other genes are shown with white arrows. 
four contained five, one contained six, and another one seven $N C R$ genes, suggesting hot spots for gene duplications in certain regions (Table 1).

\section{Nodule specificity is a common feature for all $G R P$ and $N C R$ genes.}

In order to confirm that the newly predicted GRP and $N C R$ genes without EST are indeed expressed genes, we tested their transcriptional activity by reverse transcription-polymerase chain reaction (RT-PCR) and defined their expression pattern. RNA was isolated from different $M$. truncatula organs, root, mature nodule, leaf, stem, and flower, and cDNA samples were synthesized. Specific oligonucleotides were designed for each of the 10 new MtnodGRP genes (MtnodGRP1D, MtnodGRPE, MtnodGRPF, MtnodGRPG, MtnodGRPI; MtnodGRP2C; MtnodGRP3C; MtnodGRP5B; MtnodGRP6A, and

Table 1. Genomic organization of GRP and NCR clusters

\begin{tabular}{|c|c|c|c|c|c|}
\hline Contig name ${ }^{a}$ & Genes & Orientations in contig & ORF coding exons & BAC clones & Chromosome \\
\hline MtnodGRP1A/C/D/E/E/F/G/H/I/J/K/L & 11 & $+/+/+/+/+/+/-/+/+/-/+$ & $2 / 3 / 2 / 2 / 2 / 2 / 2 / 2 / 2 / 2 / 2$ & $\begin{array}{l}\text { mth2-24k1 } \\
\text { mth2-174i23 } \\
\text { mth2-170k9 }\end{array}$ & 5 \\
\hline MtnodGRP2A/B/C $/ \mathrm{D}$ & 4 & $+/+/+/+$ & $2 / 2 / 2 / 2$ & mth2-18j19 & 2 \\
\hline MtnodGRP3A/B/트 & 3 & $+/+/+$ & $2 / 4 / 2$ & mth2-8c19 & 2 \\
\hline MtnodGRP4 & 1 & + & 2 & mth2-28h7 & 0 \\
\hline MtnodGRP5A/B & 2 & $+/+$ & $2 / 2$ & mth2-28b21 & 2 \\
\hline MtnodGRP6A/B & 2 & $+/+$ & $2 / 2$ & mth2-16818 & 2 \\
\hline NCR010/111/303 & 3 & $+/+/+$ & $2 / 2 / 2$ & mth2-5j8 & 4 \\
\hline NCR012 & 1 & + & 2 & mth2-101h21 & 1 \\
\hline NCR019 & 1 & + & 2 & mth2-167a19 & 5 \\
\hline NCR020/147 & 2 & $+/-$ & $1 / 1$ & mth2-67a20 & 7 \\
\hline NCR021 & 1 & + & 2 & mth2-59j17 & 7 \\
\hline NCR024/026/088/138/316/ $\underline{334}$ & 6 & $+/+/+/+/+/+$ & $2 / 2 / 2 / 2 / 2 / 2$ & mth2-12a23 & 2 \\
\hline NCR028 & 1 & + & 2 & mth2-62p5 & 7 \\
\hline NCR043 & 1 & + & 1 & mth2-39k21 & 4 \\
\hline NCR053/054/056/057/317 & 5 & $+/+/+/+/+$ & $2 / 2 / 2 / 2 / 2$ & mth2-35n12 & 1 \\
\hline NCR062/074/076/128/145/ $\underline{312 / 313}$ & 7 & $+/-/+/+/+/-/+$ & $2 / 2 / 2 / 2 / 2 / 2 / 2$ & mth2-65c4 & 4 \\
\hline NCR065 & 1 & + & 2 & mth2-10p14 & 2 \\
\hline NCR068 & 1 & + & 2 & mth2-175n24 & 6 \\
\hline NCR077 & 1 & + & 2 & mth2-68d12 & 3 \\
\hline NCR086/136/165/301/314 & 5 & $+/+/+/+/+$ & $2 / 2 / 2 / 2 / 2$ & mth2-10a20 & 4 \\
\hline NCR090/217/290/304/ $\underline{\underline{315}}$ & 5 & $+/-/-/-/-$ & $2 / 2 / 2 / 2 / 2$ & $\begin{array}{l}\text { mth2-15h3 } \\
\text { mth4-42c9 } \\
\text { mte1-10p15 }\end{array}$ & 5 \\
\hline NCR093 & 1 & + & 2 & mth2-101o18 & 0 \\
\hline NCR094 & 1 & + & 2 & mte1-26c9 & 5 \\
\hline NCR096 & 1 & + & 2 & mth2-10c8 & 3 \\
\hline NCR101/336 & 2 & $+/-$ & $2 / 2$ & mth2-16d16 & 3 \\
\hline NCR103/221 & 2 & $+/+$ & $2 / 2$ & $\begin{array}{l}\text { mth2-7h21 } \\
\text { mth2-116e22 }\end{array}$ & 3 \\
\hline NCR118/ㅍ18/ $\underline{319}$ & 3 & $+/+/-$ & $2 / 2 / 2$ & mth2-24k24 & 4 \\
\hline NCR119/323/324/ $\underline{335}$ & 4 & $+/+/++$ & $1 / 1 / 1 / 1$ & mth2-34p9 & 4 \\
\hline NCR121/ $\underline{320}$ & 2 & $+/+$ & $2 / 2$ & mth2-63h19 & 2 \\
\hline NCR122 & 1 & + & 2 & mth2-17e17 & 6 \\
\hline NCR127/187 & 2 & $+/-$ & $2 / 2$ & mth2-11o4 & 4 \\
\hline NCR129 & 1 & + & 2 & mth2-9k5 & 4 \\
\hline NCR140 & 1 & + & 2 & mth2-34b22 & 4 \\
\hline NCR144/266 & 2 & $+/-$ & $2 / 2$ & mth2-6h11 & 5 \\
\hline NCR146/ $\underline{321 / 322}$ & 3 & $+/+/+$ & $2 / 2 / 2$ & mth2-7m17 & 5 \\
\hline NCR $159 / 172 / 200 / 329$ & 4 & $+/+/+/+$ & $2 / 2 / 2 / 2$ & mth2-130k11 & 6 \\
\hline NCR169 & 1 & + & 2 & mth2-5n2 & 7 \\
\hline NCR171 & 1 & + & 2 & mth2-74f16 & 8 \\
\hline NCR173 & 1 & + & 1 & mth2-15117 & 2 \\
\hline NCR175/330/331 & 3 & $+/+/+$ & $2 / 2 / 2$ & mth2-32m22 & 5 \\
\hline NCR176/192 & 2 & $+/-$ & $2 / 2$ & mth2-161g1 & 0 \\
\hline NCR181 & 1 & + & 3 & mth2-50j5 & 3 \\
\hline NCR189 & 1 & + & 2 & mth2-116m2 & 3 \\
\hline NCR201/206/332/ $\underline{333}$ & 4 & $+/+/++$ & $3 / 3 / 2 / 2$ & mth2-15e11 & 4 \\
\hline NCR209 & 1 & + & 2 & mth2-31b9 & 4 \\
\hline NCR224/228/310 & 3 & $+/-/+$ & $2 / 2 / 2$ & mth2-58d22 & 6 \\
\hline NCR237/337 & 2 & $+/+$ & $1 / 1$ & mth2-20k24 & 7 \\
\hline NCR $239 / \underline{325} / 326 / 327 / 328$ & 5 & $+/+/+/+/+$ & $1 / 1 / 1 / 1 / 1$ & mth2-192a14 & 7 \\
\hline$\mathrm { NCR } 2 4 4 \longdiv { \underline { 3 3 8 } }$ & 2 & $+/+$ & $2 / 2$ & mth2-28p22 & 5 \\
\hline NCR263 & 1 & + & 2 & mth2-59b18 & 3 \\
\hline NCR265 & 1 & + & 2 & mth2-58c3 & 5 \\
\hline NCR278 & 1 & + & 2 & mth2-9f10 & 4 \\
\hline NCR284 & 1 & + & 2 & mth2-59k24 & 3 \\
\hline NCR286 & 1 & + & 2 & mth2-8d23 & 5 \\
\hline NCR289/339/340 & 3 & $+/+/+$ & $2 / 2 / 2$ & mth2-4g23 & 7 \\
\hline NCR300 & 1 & + & 2 & mth1-64n13 & 0 \\
\hline NCR309 & 1 & + & 2 & mth2-116h6 & 4 \\
\hline
\end{tabular}

${ }^{a}$ Genes underlined possess no expressed sequence tags. The names of the contigs do not reflect their real gene order. 
MtnodGRP6B) possessing no EST and for 12 new NCR genes without EST (NCR312 to NCR31318; NCR320 to NCR324) and were used for PCR experiments (Fig. 2). The Mtc27 constitutive marker was used as control for cDNA quality. PCR products were then cloned and sequenced to distinguish the highly homologous genes and confirm their RNA splicing prediction. All investigated genes were transcribed, our splicing predictions were correct, and these genes exhibited exclusive nodule-specific expression (Fig. 2), as it was found for the previously identified GRP and NCR genes (Kevei et al. 2002; Mergaert et al. 2003).

\section{Gene organization in $G R P$ clusters.}

Most MtnodGRP genes are composed of two exons where the first exon (approximately $80 \mathrm{bp}$ ) codes for the signal peptide and the second exon (between 200 and $700 \mathrm{bp}$ ) for the glycinerich domain. Two exceptions were found: MtnodGRP1C had two introns, while the longer MtnodGRP3A was encoded by four exons. Intron sequences of the GRP genes were not homologous. In the different GRP contigs, all GRP genes were in the same orientation, except MtnodGRPIH and $K$, which were in opposite orientation to the others (Fig. 1).

Protein homology levels within the GRP gene clusters were investigated to elucidate the duplication events that occurred in the different contigs. Two examples are shown in Figure 3. In the MtnodGRP2 contig, the homology among the four MtnodGRP2 proteins was obvious; however, certain members were more similar to each other than to others, and divergence of the four proteins was predominant in the C-terminus (60 to $80 \%$ similarity). MtnodGRP1A, MtnodGRP1D, MtnodGRP1E, MtnodGRPIF, and MtnodGRPIG genes coded also for homologous proteins, but the identity was more apparent for the signal peptide domain (90\% similarity). The homology and the same orientation of MtnodGRP2 and these MtnodGRP1 genes suggest that these genes had arisen from tandem duplication events within a restricted DNA region. In addition to the closely located MtnodGRP1A, MtnodGRP1D, MtnodGRP1E, MtnodGRPIF, and MtnodGRPIG genes, the MtnodGRPl contig contained six other GRP (MtnodGRP1C, MtnodGRP1H, MtnodGRP1I, MtnodGRP1J, MtnodGRP1K, MtnodGRP1L) that were more dispersed on the contig (Fig. 1). In these latter MtnodGRP1 proteins, the signal peptides were similar but the glycine-rich domains were divergent. Thus, the duplication events resulting in the MtnodGRPIC, MtnodGRPIH, MtnodGRP1I, MtnodGRP1J, MtnodGRPIK, and MtnodGRPIL genes occurred earlier than the formation of the MtnodGRPIA, MtnodGRP1D, MtnodGRPlE, MtnodGRPIF, and Mtnod$G R P 1 G$ subgroup. This prediction is also supported by the insertions of numerous genes and by the larger distances between the presumably more ancient MtnodGRP1 genes.

The phylogenetic comparison of the 23 MtnodGRP proteins divided them in two major groups separating most MtnodGRP1 proteins (MtnodGRP1A, MtnodGRP1C, MtnodGRP1D, MtnodGRP1E, MtnodGRP1F, MtnodGRP1G, MtnodGRP1L) from the others (Fig. 4). On the other branch, MtnodGRP2, MtnodGRP5, and MtnodGRP6 proteins were closely related. Despite the gene cluster structures, some GRP showed higher homology to GRP present in other contigs, as was the case for MtnodGRP1J, which was more related to MtnodGRP3A, MtnodGRP3B, and MtnodGRP4 than to the other MtnodGRP1 proteins. MtnodGRP3C also displayed a closer relation to MtnodGRP6 proteins than to the other MtnodGRP3. MtnodGRP1H, MtnodGRP1I, and MtnodGRP1K were more distant from the other GRP as well as the previously identified nodulin MtN29 (Gamas et al. 1996), which also belongs to the nodule-specific GRP (Kevei et al. 2002) but represents a distant member. In conclusion, the evolutionary history of the GRP family is complex with ancient and more recent duplications that occurred both locally and at a long range between chromosomes.

\section{$N C R$ genes and their cluster organization.}

Similarly to GRP, most of the identified $N C R$ genes were composed of two open reading frame (ORF) coding exons. The first coded for the signal peptide (approximately $70 \mathrm{bp}$ ) and the second for the mature peptide (approximately $150 \mathrm{bp}$ ). The average intron length was 100 to $150 \mathrm{bp}$. Nevertheless, in a few cases $N C R$ contained only one ORF coding exon (Table 1). Another uncharacteristic NCR gene structure consisted in three exon-containing genes (Table 1). In NCR201 and $N C R 206$, the third exon coded for the last seven and six amino acids, respectively. In the NCR181 gene, the second exon was small, coding for eight amino acids and the cysteine-rich domain derived from the third exon. In the NCR clusters, the genes were usually in the same orientation (Table 1). In total, 24 contigs contained from two to seven NCR genes, and they were clustered within distinct lengths of genomic regions from 7 up to $364 \mathrm{~kb}$.

The homology levels of NCR within their clusters were variable, as shown for three of them in Figure 3. Members of the

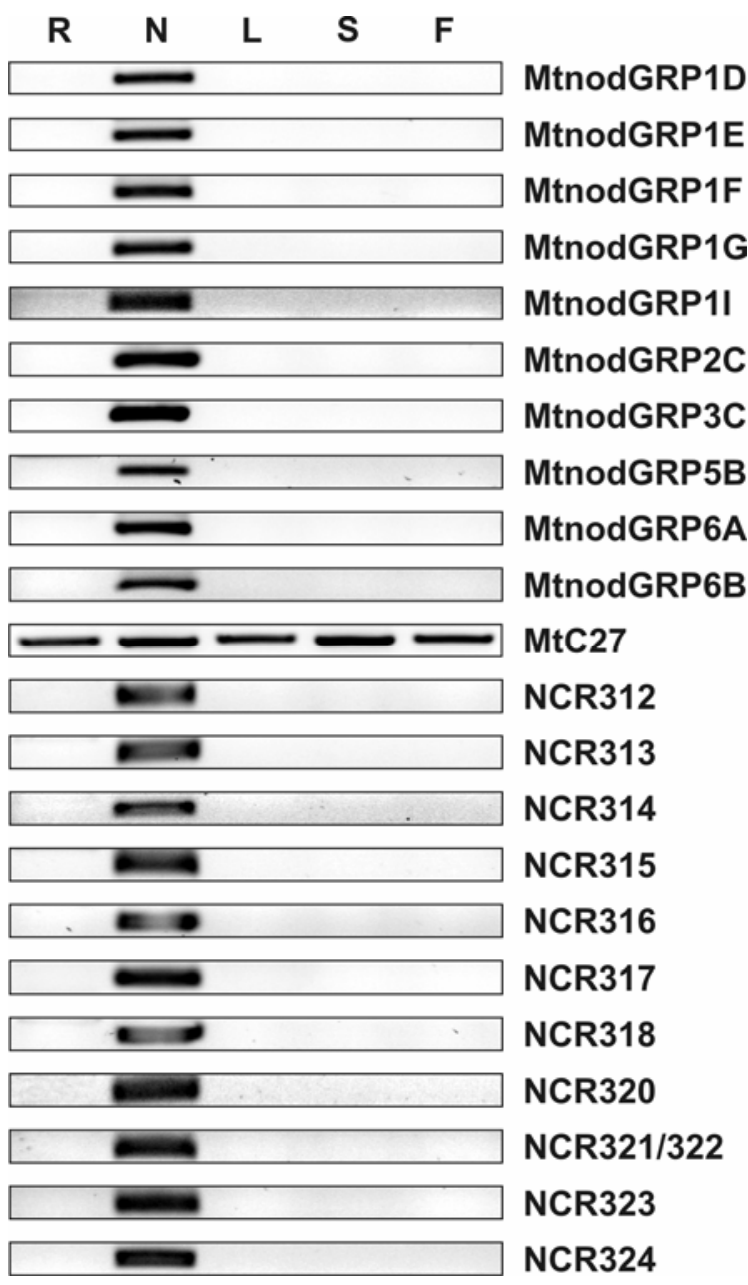

Fig. 2. Expression pattern of new GRP and $N C R$ genes determined by reverse transcription-polymerase chain reaction (RT-PCR) in different tissues of Medicago truncatula. RT-PCR reactions were performed on cDNA prepared from roots $(\mathrm{R})$, nodules $(\mathrm{N})$, leaves $(\mathrm{L})$, stems $(\mathrm{S})$, and flowers (F). In the case of NCR321 to NCR 322, primers could amplify products of similar sizes for both genes and sequencing the products allowed us to distinguish them. Amplification of $M t C 27$ was used as a positive control for each condition. 
NCR159/172/200/329 cluster were highly homologous (74\% average similarity), both on the signal peptide and on the mature parts of the proteins. In the NCR239/325/326/327/328 cluster, NCR325 and NCR326 shared 90\% similarity, having a practically identical signal peptide and a few differences in the mature domain. In contrast, NCR239, NCR327, and NCR328 were less homologous (64\% average similarity) and likely arose from earlier duplications than the NCR325 and NCR326 genes. The divergence in the NCR090/219/290/304/315 cluster was even more pronounced (55\% average similarity), affecting the entire peptide and particularly the mature part, in which only the position of the cysteines and a few neighboring amino acids were conserved. In respect to all investigated clusters of the NCR family (Fig. 5), the position of the cysteines was conserved and the N-terminal signal peptide part was significantly more homologous than the $\mathrm{C}$-terminal region.

The evolutionary relatedness of the 108 NCR by phylogenetic analysis is given in Figure 5. Generally the NCR that were present in the same cluster were the most homologous and resulted from the most recent duplication events (Fig. 5, shaded boxes). However, others present in the same cluster, like NCR090/217/290/304/315, were distantly related and had arisen from more ancient duplication events permitting extensive diversifications during the evolution (Fig. 5, asterisks).

The GRP and NCR genes on Medicago chromosomes.

In addition to the cluster analysis, the GRP and NCR gene organizations were investigated on the whole-genome level. The chromosome visualization tool from the Medicago truncatula website was used to determine the gene or BAC positions on different chromosomes of M. truncatula (Fig. 6).

Of the six contigs containing the 23 GRP genes, four were on chromosome 2 and one on chromosome 5, while the MtnodGRP4 gene was on chromosome 0 . The three contigs containing the most related GRP, MtnodGRP2, MtnodGRP5 and MtnodGRP6 (Fig. 4), were also closely linked on chromosome 2.

Unlike the GRP, the 51 NCR loci were dispersed throughout the entire genome. Certain regions of chromosomes 3, 4, and 5
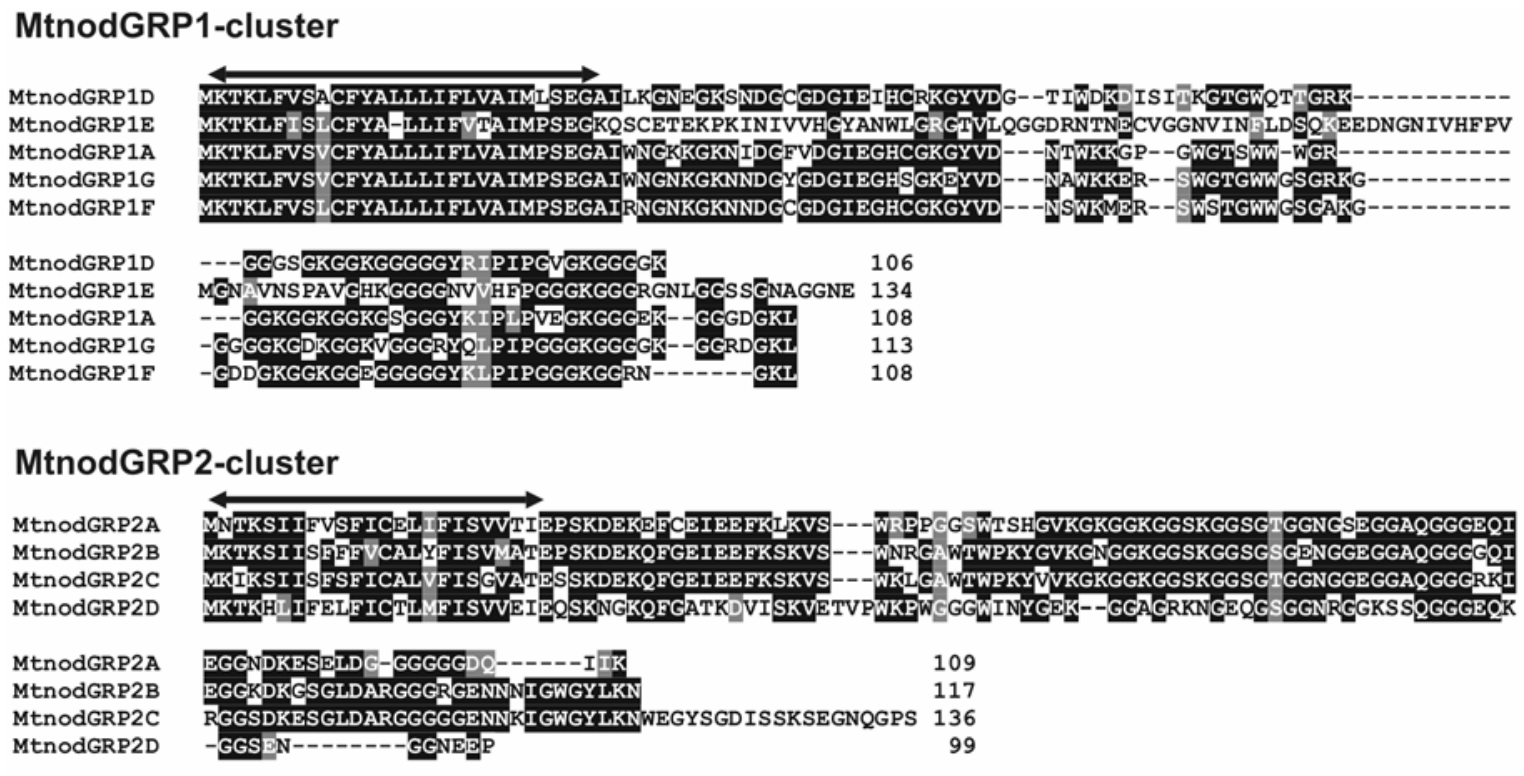

NCR159/172/200/329-cluster

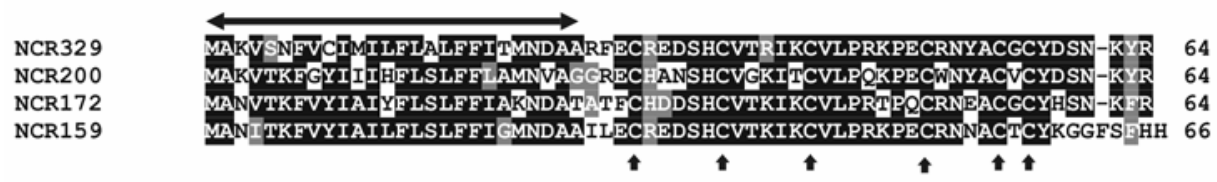

NCR239/325/326/327/328-cluster

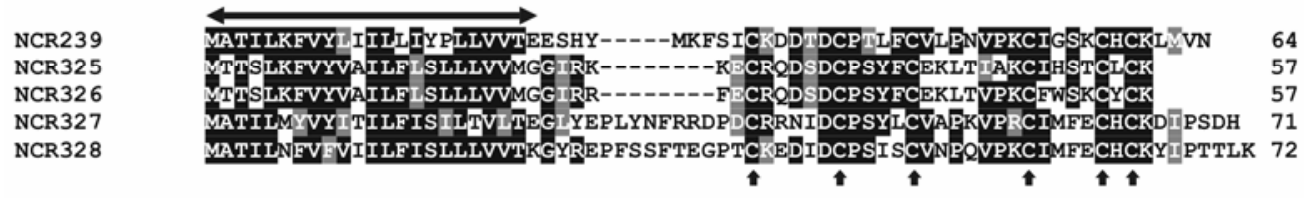

NCR090/217/290/304/315-cluster

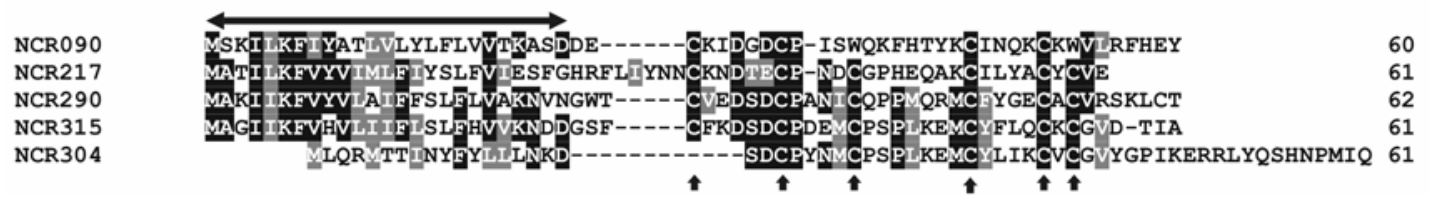

Fig. 3. Peptide alignment of two representative GRP clusters and three representative NCR clusters. Not all the sequences from each cluster are included. Identical residues are shaded black, while similar residues are shaded gray. The big arrows delimit the signal peptide part of the proteins. The small arrows show the cysteines in conserved positions. 
possessed numerous $N C R$ genes, while a single gene was present on chromosome 8 , and two loci were present on chromosome 1. Nevertheless, the Medicago sequencing project is still ongoing, and therefore, our results are not exhaustive and it is possible that additional $N C R$ will be found. Interestingly, in a region on chromosome 2, the NCR024/026/088/138/316/334 cluster and the MtnodGRP3 genes were in the same contig. So far, we have no evidence for other genomic regions containing closely linked GRP and NCR genes, but in the absence of the complete genome sequence, we cannot exclude that either.

\section{Gene evolution of $G R P$ and $N C R$.}

The comparison of GRP and NCR in Figure 3 indicates that the signal peptide and mature peptide part of the proteins might diversify to different extents. Their evolution patterns were separately analyzed by the ratios of synonymous (dS) and nonsynonymous (dnS) amino-acid substitution; a value for $\mathrm{dS} / \mathrm{dnS}>1$ indicates purifying and $\mathrm{dS} / \mathrm{dnS}<1$ indicates diversifying selection for the investigated domains. All 23 GRP in the six clusters and 37 NCR of the NCR090, NCR159, NCR239, NCR053, NCR086, NCR024, and NCR062 clusters (Table 1) were used for the analysis. In the case of the GRP, $60 \%$ of the signal peptide pairwise comparisons (PC) fall into the $\mathrm{dS} / \mathrm{dnS}>1$ category of purifying selection (Fig. 7A), while glycine-rich domains followed diversifying evolution $(88 \%$ of PC with dS/dnS < 1) (Fig. 7B). In the 37 studied NCR, 55\% of the signal peptide PC showed purifying selection (Fig. 7C), while $91 \%$ of the cysteine-rich domains PC (Fig. 7D) showed evidence for diversifying selection. Thus the diversification of the mature peptides was even more pronounced in the NCR. However, this statement concerns the overall comparison, and the $\mathrm{dS} / \mathrm{dnS}$ values were variable depending on the NCR cluster and the time of the gene duplication events. Naturally, the more recently duplicated genes had less chance for diversification.

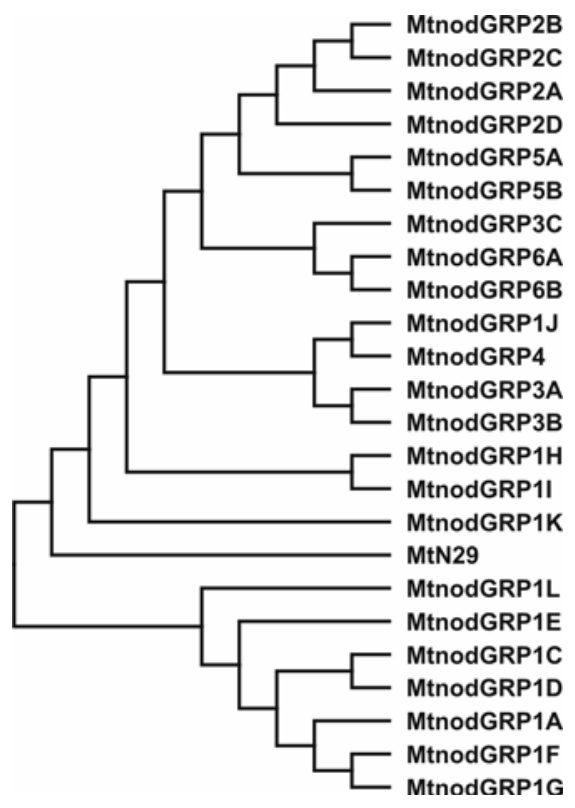

Fig. 4. Phylogenetic relationship between GRP proteins. All GRP except MtN29 have available genomic sequences.

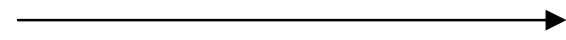

Fig. 5. Phylogenetic relationship between NCR peptides. Only NCR for which the corresponding genomic sequence has been identified are included in this tree. Shaded boxes show homologous genes that are clustered in the genome. NCR marked with an asterisk form a cluster in the genome but are not homologous.

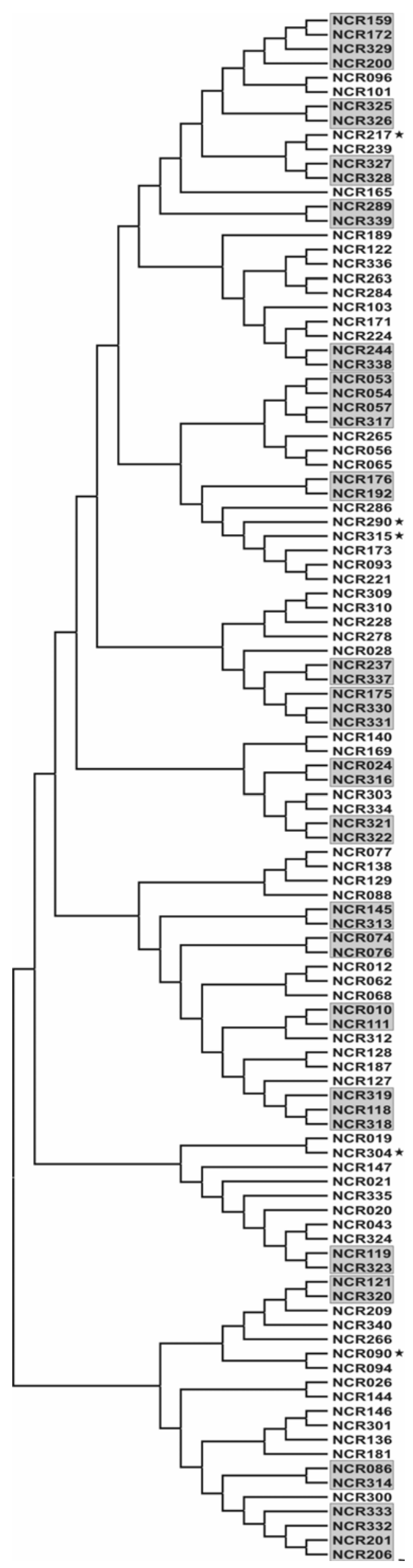

Vol. 20, No. 9, 2007 / 1143 
Overall, these results show, for the GRP and NCR, a tendency of purifying selection for their signal peptides contrasting with the diversifying selection trend observed for their respective glycine-rich or cysteine-rich domains.

\section{Microsyntenic analysis of $G R P$ and $N C R$ loci.}

In the L. japonicus databases, there are no ESTs or genomic sequences corresponding to $M$. truncatula GRP and NCR. Therefore, we carried out microsynteny analysis in the two model legumes to verify whether the GRP and NCR genes are unique to $M$. truncatula. Annotated $M$. truncatula protein sequences corresponding to the flanking genomic regions of different GRP and NCR gene clusters were used for tBLASTn searches against $L$. japonicus genome databases. The corresponding L. japonicus regions were found for the $M$. truncatula loci carrying the MtnodGRP2 and the NCR062/074/076/ 128/145/312/313 clusters (Fig. 8). Analysis of gene occurrence and order in the two genomes demonstrated a syntenic relation between $M$. truncatula and L. japonicus in these regions.

In the MtnodGRP2 region on chromosome 2, nine of 17 genes showed synteny to eight of nine genes of the L. japonicus chromosome 6 (Fig. 8). However, the four tandem-duplicated MtnodGRP2A, MtnodGRP2B, MtnodGRP2C, and Mtnod$G R P 2 D$ genes were absent in $L$. japonicus. In the syntenic region, all genes except one were in the same orientation in the two genomes and the syntenic proteins displayed, on average, $75 \%$ identity and $84 \%$ similarity. In the case of the NCR062/074/076/128/145/312/313 contig on M. truncatula chromosome 4, 10 of 38 genes displayed syntenic relationship with 10 of 19 L. japonicus genes on chromosome 3 . All the syntenic genes were in the same orientation in the two genomes, with one exception, and the encoded proteins shared an average of $81 \%$ identity and $92 \%$ similarity. Similarly to the MtnodGRP2 contig, no NCR-like genes were present in the corresponding region on L. japonicus chromosome 3. However, unlike the MtnodGRP 2 region, many other genes were inserted between the syntenic genes and the M. truncatula NCR genes. In addition, the duplication events resulting in NCR312 and NCR074 genes were associated with gene inversion. These data suggest that the MtnodGRP2 gene duplications occurred later than the amplifications of these $N C R$.
In the absence of complete genome sequences, our analysis was restricted to the available two examples. Nevertheless, this set of data, as well as the absence of GRP and NCR EST in $L$. japonicus and Glycine max but the presence of GRP and NCR transcripts in other IRLC legumes (Kevei et al. 2002; Mergaert et al. 2003) reinforce the idea that these gene families are indeed specific for IRLC legumes.

\section{DISCUSSION}

\section{$N C R$ and $G R P$ gene families in M. truncatula.}

$N C R$ genes likely represent one of the largest multigenic families in plants with extreme natural diversity in the mature peptides. From the $311 N C R$ transcripts described by Mergaert and associates (2003), in this study, we localized the genomic loci for 79 genes, representing $25 \%$ of the known set in $M$. truncatula. Moreover, we identified 29 new NCR in the vicinity of known $N C R$, suggesting that the family is much larger than expected. One has to note that all verified new $N C R$ were expressed genes and displayed nodule specificity as shown by RT-PCR experiments. Extrapolating our findings to the wholegenome level would predict a family size of about 430 nodulespecific NCR genes. Thus, our strategy to find new NCR family members was fruitful despite the fact that bioinformatics tools are not optimized to detect short and divergent genes, as discussed by Silverstein and associates (2005).

Until this study, many GRP remained hidden and were not annotated in the genome due to their high variability and lack of apparent glycine-rich motifs in the mature peptide. As in the case of NCR, we were able to identify 17 novel genes, mainly on the basis of the highly conserved signal peptide. At present, 23 GRP belonging to six subgroups are known in M. truncatula.

\section{Genome organization and evolutionary dynamics of $N C R$ and $G R P$ gene families.}

Few NCR and GRP exist as single genes in a locus, but they are mostly clustered with multiple genes per locus. Their genomic organizations and evolutionary scenario with the rapid diversification by local duplications are features that are also common in the innate immunity gene families such as $R$

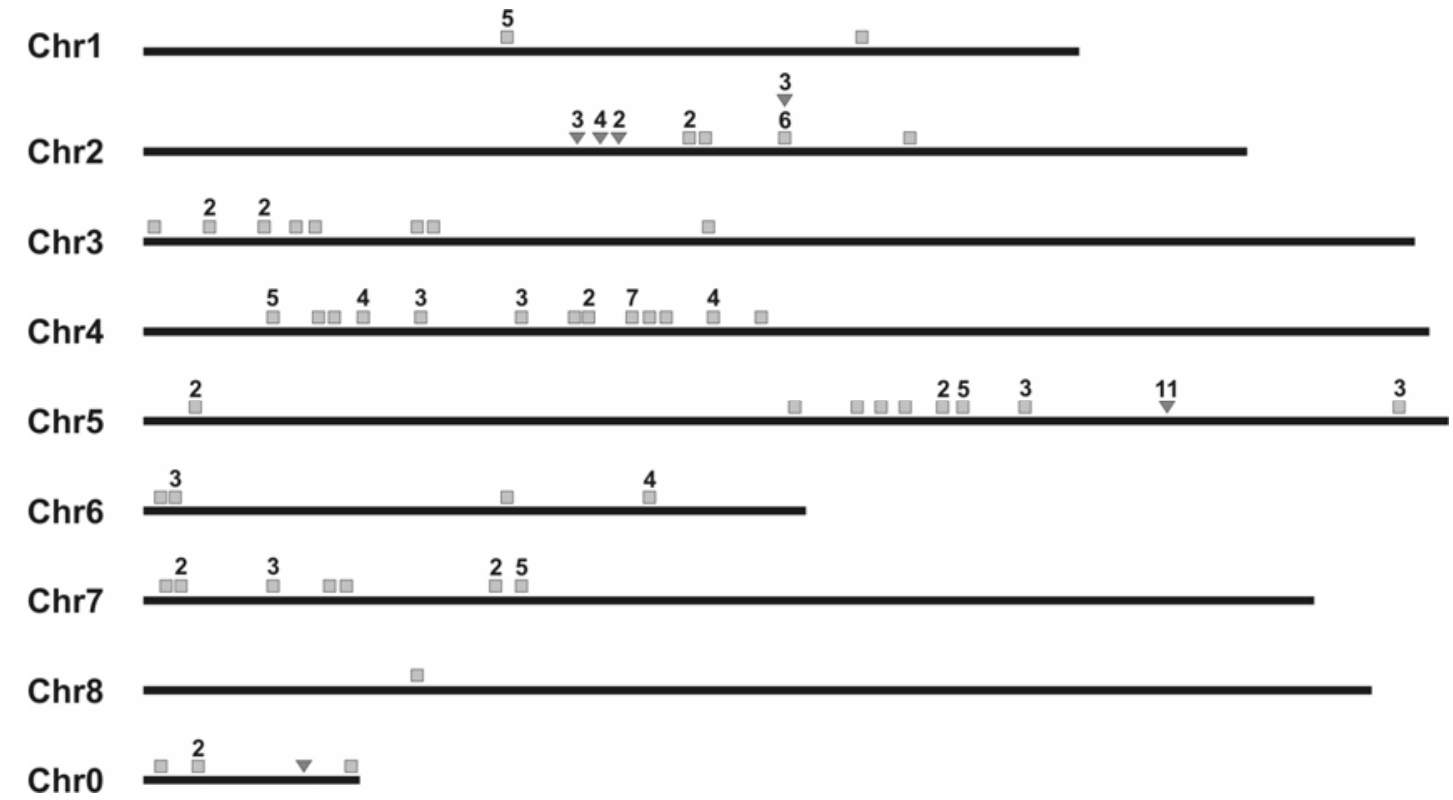

Fig. 6. Genome scale organization of GRP and NCR genes in Medicago truncatula. NCR loci are indicated by squares, whereas GRP loci are represented by triangles on the pseudochromosomes according to the chromosome visualization tool from the Medicago truncatula website. The number of genes per locus is indicated when more than one gene could be identified in the contig. 
genes and defensins (Baumgarten et al. 2003; Meyers et al. 2003; Silverstein et al. 2005). These defensins are also cysteine-rich secreted peptides and members of the CCP family, with their eight cysteines forming four disulfide bridges (Shutte et al. 2002). As observed by dS/dnS aminoacid substitution rates analysis, the signal peptide of defensins displays purifying selection, whereas the mature peptide evolution is driven by diversifying selection (Maxwell et al. 2003; Semple et al. 2003; Silverstein et al. 2005).

Similarly to defensins, the cysteine-rich regions of NCR and the glycine-rich domains of GRP undergo diversifying selection. The evolution of signal peptides is less evident, though there is a tendency for purifying selection in both peptide families. For the recently evolved and highly similar genes, such as those in the MtnodGRP2 or NCR159/172/200/329 clusters, we cannot draw conclusions with certainty for their future evolution, but we presume that they will follow a similar evolutionary scenario as the other genes of the family.

A

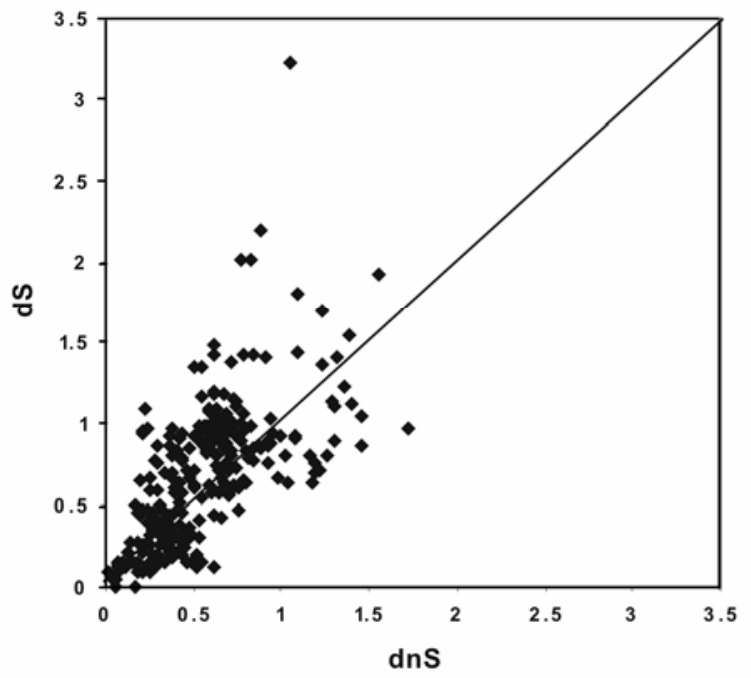

C

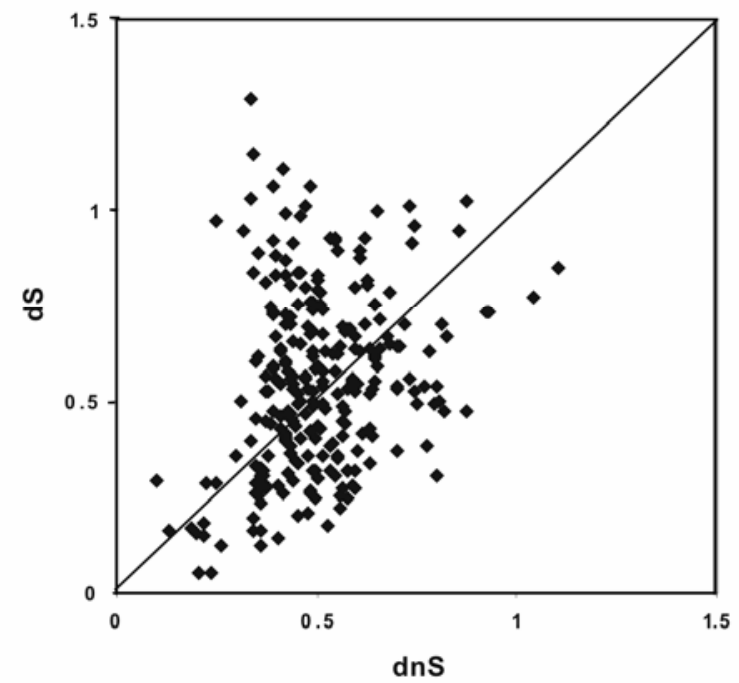

GRP and NCR families are unique to IRLC legumes.

Besides the nodule specificity of the GRP and NCR gene families, the expression data suggested that they were restricted to IRLC legumes like Medicago spp. (Fedorova et al. 2002; Graham et al. 2004; Györgyey et al. 2000; Mergaert et al. 2003), Pisum sativum (Kardailsky et al. 1993; Kato et al. 2002; Scheres et al. 1990;), Trifolium repens (Crockard et al. 2002), Astragalus sinicus (Chou et al. 2006), Galega orientalis (Kaijalainen et al. 2002), and Vicia faba (Frühling et al. 2000), in which related sequences have been identified during EST sequencing projects or searches for nodule-enhanced or nodule-specific genes. In spite of the large number of EST available from other distantly related legume species, like Glycine max, L. japonicus, or Phaseolus vulgaris, no homologs of GRP or NCR were found. In our study, microsyntenic evidences reinforced the hypothesis that $G R P$ and NCR are unique to IRLC legumes, since they were absent in the syntenic L. japonicus regions. Thus, these two families should have appeared between 51 and 25 million years ago,

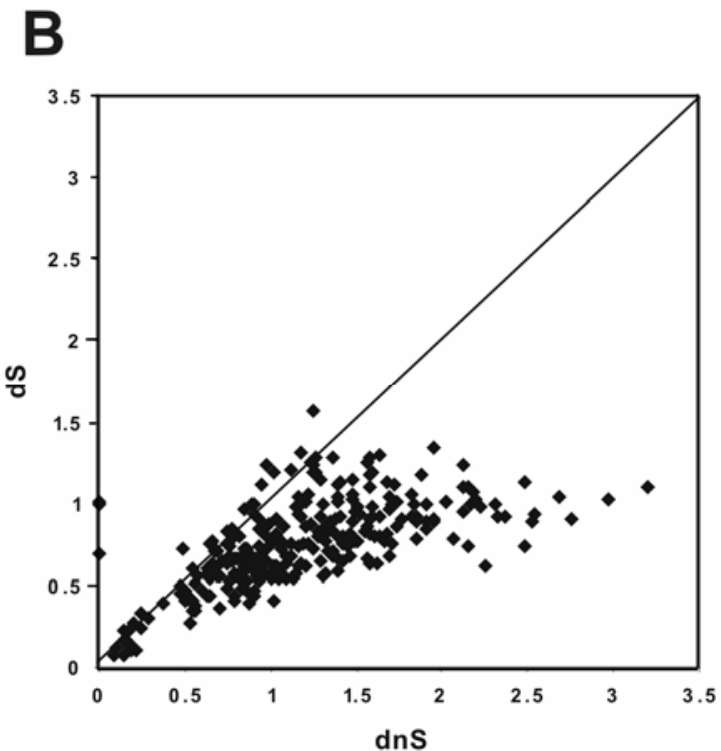

D

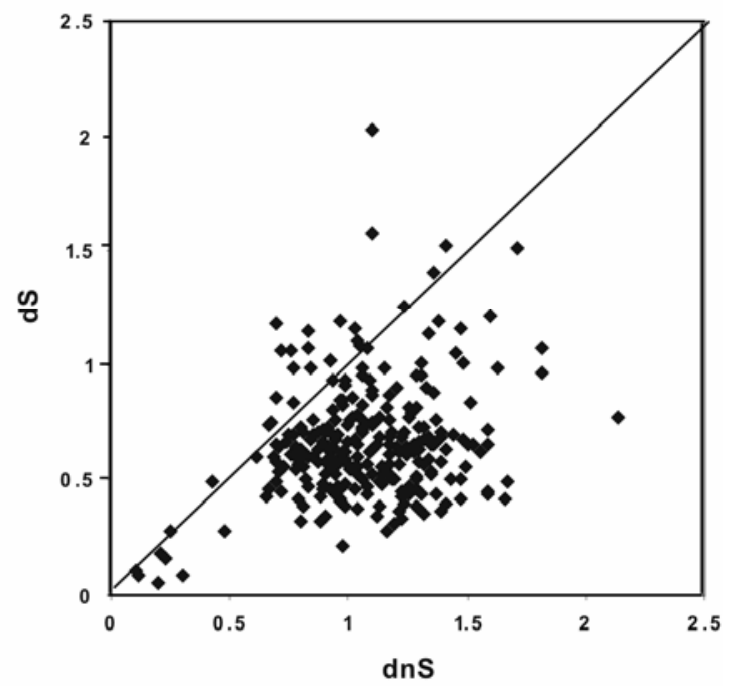

Fig. 7. Plot of synonymous (dS) versus nonsynonymous $(\mathrm{dnS})$ substitution rates for 23 GRP and 37 NCR in Medicago truncatula. The signal peptide (A, GRP; C, NCR) and mature peptide (B, GRP; D, NCR) sequences were analyzed separately. The $x$ axis shows the dnS values, the $y$ axis the dS values. Every point corresponds to the pairwise comparison between two nucleotide sequences. The diagonal line $(\mathrm{dS} / \mathrm{dnS}=1)$ represents the neutral selection. Points above this line $(\mathrm{dS} / \mathrm{dnS}>1)$ marked purifying selection, points under the line $(\mathrm{dS} / \mathrm{dnS}<1)$ displayed diversifying selection. 
which corresponds, respectively, to the estimated times for the most recent common ancestor (MRCA) of Lotus, Pisum, and Medicago and the age of separation of the MRCA of Medicago and Pisum, respectively (Lavin et al. 2005). The availability of nodule EST databases from other IRLC legumes would be beneficial to elucidate the history of these gene families and decipher how rapidly they became diverged among the IRLC plants. One can postulate that these gene families had already started to diversify before the Medicago-Pisum separation, as most NCR-containing IRLC plants harbor several members of the gene family. Also, phylogenetic analysis of NCR originating from different IRLC species shows higher homology between members of different IRLC species than to other members from the same organism (data not shown). These results indicate that the radiation of this gene family has probably started before the successive speciation events that lead to the actual diversity of the IRLC legumes. In other words, the MRCA of IRLC legumes should have already had $N C R$ in its genome. Despite the limited data set available for the smaller $G R P$ family, the same evolutionary scenario can be predicted, as $G R P$ have, so far, been found exclusively in IRLC legumes. Thus GRP and NCR families should have appeared almost simultaneously during legume diversification.

\section{What are the functions for so many genes?}

$G R P$ form a family with at least 23 genes and the NCR family is composed of up to more than 400 genes, while up to $5 \%$ of EST databases from Medicago nodules are composed of NCR. Yet, no function has been assigned to these two gene families. Mergaert and associates (2006) proposed that NCR, like defensins, could act as antimicrobial peptides and may govern bacteroid differentiation. This hypothesis is in agreement with the differences in morphology and physiology of the bacteroids in IRLC and non-IRLC legumes (Mergaert et al. 2006). In IRLC legumes, bacteroids undergo a multistep irreversible differentia- tion process manifested by loss of cell division ability and a striking elongation of the cells that is driven by endoreduplication cycles. These modifications are accompanied by membrane permeabilization and the inability of bacteroids to recover growth once they were released from the nodule. In contrast, in non-IRLC legumes, bacteroids are able to divide and resume growth when released from a nodule. They have no cell elongation, genome amplification, or membrane permeabilization. We have shown previously that the observed differences in bacteroid behavior are due to plant factors and not to the bacterial genetic background. This suggests a role for IRLC-specific genes like $N C R$ and GRP that may act as antimicrobial peptides (AMP) especially recruited for bacteroid differentiation. The known AMP are relatively small $(<10 \mathrm{kDa})$, cationic and amphipathic peptides of variable length and structure (Brogden 2005). Most AMP are believed to act by disrupting the plasma membrane integrity leading to cell lysis. In plants, the main groups of AMP are cysteine-rich defensins. NCR are also in the CCP group like the defensins but are expressed only in the Rhizobium-infected nodule cells in which they might have specific functions in bacteroid differentiation, for example, leading to increase membrane permeability and inhibiting cell division. AMP can be rich in other amino acids as well, such as glycines (Ötvös 2000). Thus, GRP could also be considered as a specific type of AMP acting in symbiosis. AMP act often synergistically. This could explain overlapping expression of several NCR and GRP in the same nodule cell. Moreover, peptides are often involved in cellto-cell communications. As the different NCR and GRP are expressed in the different stages of symbiotic cell differentiation, they could act, not simply as selective bacteriostatic agents controlling the microsymbionts inside the nodule, but could also monitor differentiation of bacteroids in accordance with the host cells. In addition, the high genetic diversity of isolates from the same rhizobial species in natural ecosystems (combined with the necessity to reinitiate the symbiosis at each generation of the

\section{MtnodGRP2-contig (mth2-18j19) - chr2}

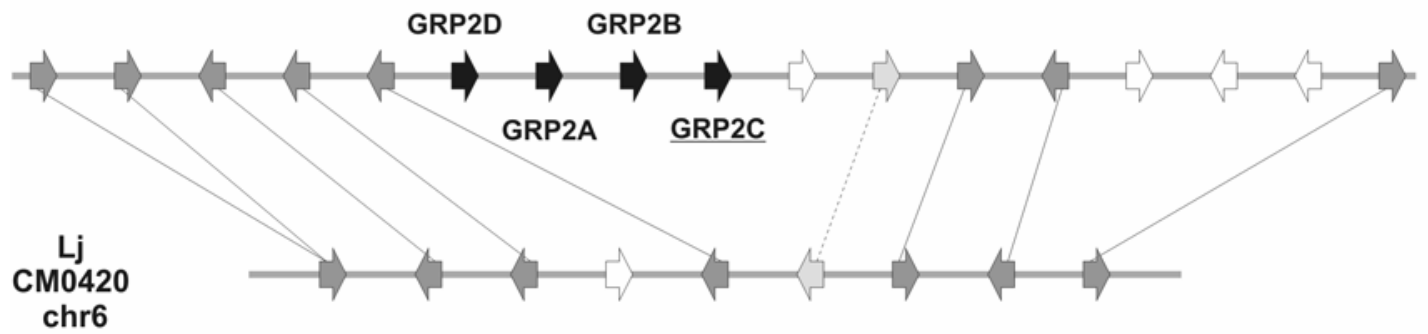

NCR062/074/076/128/145/312/313-contig (mth2-65c4,mth2-94j16) - chr 4

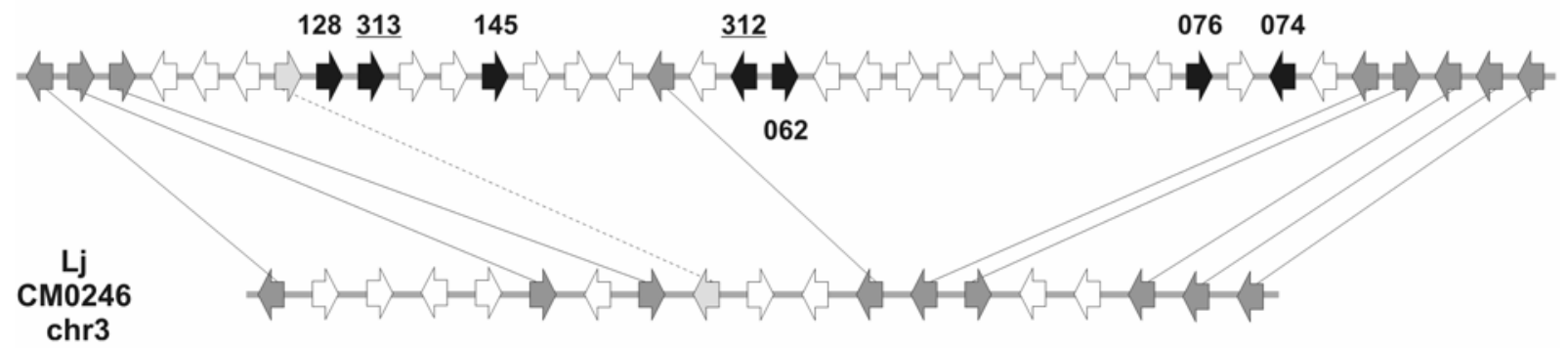

Fig. 8. Microsyntenic comparison between Medicago truncatula and Lotus japonicus on MtnodGRP2 and NCR062/074/076/128/145/312/313 contigs. The BAC clone numbers corresponding to the Medicago and Lotus contigs are indicated and followed by the chromosome number. The location and orientation of genes is indicated by arrows. Genes and intergenic regions are not drawn at scale. Black arrows represent the GRP or NCR genes and their underlining indicates the absence of expressed sequence tags. Gray linked arrows show syntenic genes with the same orientation, light gray ones show genes with inversion. Other nonsyntenic genes are shown with white arrows. 
plant) might have been an additional driving force in IRLC legumes for diversification of $N C R$ and GRP. Thereby, the legume host could be armed to confront any kind of microsymbiont isolate, to control their fate and differentiation inside the nodule. This high diversity of NCR or GRP ensures that the bacterium cannot overcome the effect of single NCR or GRP and escape the differentiation process imposed by the plant.

Functional studies of these gene families are of great interest with respect to symbiosis and bacteroid differentiation, but in addition, this high natural diversity of legume peptides might have diverse applications in human, animal, and plant healthcare.

\section{MATERIALS AND METHODS}

\section{$N C R$ and $G R P$ genes identification.}

BLASTn (Altschul et al. 1997) analyses were performed against the NCBI nonredundant database, using GRP and NCR transcripts as query (Kevei et al. 2002; Mergaert et al. 2003). M. truncatula BAC clone sequences corresponding to perfect matches with the transcripts were used in further BLASTn analyses to extend the contigs to their maximal size. These contigs were then screened for the presence of other NCR or $G R P$ using the putative mature or signal peptide sequence in a tBLASTn analysis.

\section{Protein alignments and phylogenetic analyses.}

Protein alignments shown in Figure 3 were produced using the ClustalW application of the BioEdit v6.0 pack and were processed by EMBnet's Boxshade 3.21 program. After the alignments, the predicted peptide sequences were processed by the MEGA 3.1 software (Kumar et al. 2004) to produce neighbor-joining trees of GRP and NCR.

\section{Evolutionary analysis of NCR and GRP in M. truncatula.}

In order to estimate the rates of $\mathrm{dS}$ and $\mathrm{dnS}$ substitutions, the coding sequences of NCR and GRP were split into the signal peptide and the mature peptide part according to the SignalP prediction server. The coding sequences were aligned by ClustalW. The dS and dnS substitution values were calculated by the Nei-Gojobori method (Jukes-Cantor correction), which, in addition to counting the number of $\mathrm{dS}$ and $\mathrm{dnS}$ substitutions, takes into account the multiple potential substitutions at the same place. Gaps were removed in each pairwise comparison. All results were computed by MEGA 3.1 software. All pairwise comparisons of $\mathrm{dS}$ and $\mathrm{dnS}$ values were carried out for the different domains and were used for plot analysis.

Microsynteny study between $M$. truncatula and $L$. japonicus.

Predicted protein sequences of the contigs corresponding to $G R P$ or $N C R$ clusters and neighboring regions were used in a tBLASTn analysis against a local database composed of $L$. japonicus genomic sequences previously created in BioEdit. $L$. japonicus predicted genes sharing $\geq 80 \%$ homology with $M$. truncatula predicted genes were considered as valid anchors.

\section{Gene plotting on $M$. truncatula pseudochromosomes.}

Predicted protein sequences from either GRP or NCR genes were submitted to the chromosome visualization tool on the Medicago truncatula Sequencing Resources website and the corresponding BAC hit was localized on the genetic map.

\section{RT-PCR experiments.}

Leaf, stem, flower, 21-dpi nodule, and root tissue samples of M. truncatula J5 plants were collected, and RNA was extracted, using the RNeasy plant mini kit, and DNase treated (Qiagen S.A., Courtaboeuf, France). First-strand cDNAs were synthe- sized in the presence of RNasin with SuperScript reverse transcriptase according to the manufacturer's instructions (Promega, Madison, WI, USA). Gene-specific PCR primers were designed and synthesized on the Invitrogen website. RT-PCR were performed for 35 cycles using Taq DNA polymerase (Eurogentec, Liège, Belgium) in an iCycler (BIORAD, Marnes la Coquette, France). PCR products were run on $1.5 \%$ (wt/vol) agarose gels and were cloned into pGEM-T Easy vectors (Promega) and were sequenced using BigDye Terminator v3.1 in an ABI PRISM 3100 (Applied Biosystems, Courtaboeuf, France). Sequences were then aligned with genomic sequences to verify identity and splicing prediction.

\section{ACKNOWLEDGMENTS}

We thank N. D. Young (University of Minnesota, Minneapolis, U.S.A.), S. Tabata and S. Sato (Kazusa DNA Research Institute, Chiba, Japan) for kindly providing L. japonicus genomic sequences. This work was supported by grants from the French Agence National de la Recherche Nodanti-mic project number ANR-05-BLAN-0129-01 and by the European Union FP6 Grain Legume IP no. 506223. B. Alunni was the recipient of a Ph.D. fellowship from the French Ministry of Research, and M. RedondoNieto was funded by the Spanish Ministerio de Educación y Ciencia Progama Becas Postdoctorales en España y en el extranjero 2003.

\section{LITERATURE CITED}

Altschul, S. F., Madden, T. L., Schaffer, A. A., Zhang, J., Zhang, Z., Miller, W., and Lipman, D. J. 1997. Gapped BLAST and PSI-BLAST: A new generation of protein database search programs. Nucleic Acids Res. 25:3389-3402.

Baumgarten, A., Cannon, S., Spangler, R., and May, G. 2003. Genome-level evolution of resistance genes in Arabidopsis thaliana. Genetics 165:309319.

Brogden, K. A. 2005 Antimicrobial peptides: Pore formers or metabolic inhibitors in bacteria? Nature Rev. Microbiol. 3:238-250.

Cannon, S. B., Sterck, L., Rombauts, S., Sato, S., Cheung, F., Gouzy, G., Wang, X., Mudge, J., Vasdewani, J., Schiex T, Spannagl, M., Monaghan, E., Nicholson, C., Humphray, S. J., Schoof,, H., Mayer, K. F. X., Rogers, J., Quétier, F., Oldroyd, G. E., Debelé, F., Cook, D. R., Retzel, E. F., Roe, B. A., Town, C. D., Tabata, S., Van de Peer, Y., and Young, N. D. 2006. Legume genome evolution viewed through the Medicago truncatula and Lotus japonicus genomes. Proc. Natl. Acad. Sci. U.S.A. 103:14959-14964.

Chou, M. X., Wei, X. Y., Chen, D. S., and Zhou, J. C. 2006. Thirteen nodule-specific or nodule enhanced genes encoding products homologous to cysteine cluster proteins or plant lipid transfer proteins are identified in Astragalus sinicus L. by suppressive subtractive hybridization. J. Exp. Bot. 57:2673-2685.

Crockard, A., Bjourson, J., Dazzo, B., and Cooper, J. E. 2002. A white clover nodulin gene, $d d 23 b$, encoding a cysteine cluster protein, is expressed in roots during the very early stages of interaction with Rhizobium leguminosarum biovar trifolii and after treatment with chitolipooligosaccharide Nod factors. J. Plant Res. 115:439-447.

El Yahyaoui, F., Kuster, H., Ben Amor, B., Hohnjec, N., Puhler, A., Becker, A., Gouzy, J., Vernie, T., Gough, C., Niebel, A., Godiard, L., and Gamas, P. 2004. Expression profiling in Medicago truncatula identifies more than 750 genes differentially expressed during nodulation, including many potential regulators of the symbiotic program. Plant Physiol. 136:3159-3176.

Fedorova, M., van de Mortel, J., Matsumoto, P. A., Cho, J., Town, C. D., VandenBosch, K. A., Gantt, J. S., and Vance, C. P. 2002. Genome-wide identification of nodule-specific transcripts in the model legume Medicago truncatula. Plant Physiol. 130:519-537.

Frühling. M., Albus, U., Hohnjec, N., Geise, G., Pühler, A., and Perlick, A. M. 2000. A small family of broad bean codes for late nodulins containing conserved cysteine clusters. Plant Sci. 152:67-77.

Gamas, P., de Carvalho Niebel, F., Lescure, N., and Cullimore, J. 1996. Use of a subtractive hybridization approach to identify new Medicago truncatula genes induced during root nodule development. Mol. PlantMicrobe Interact. 9:233-242.

Graham, M. A., Silverstein, K. A., Cannon, S. B., and VandenBosch, K. A. 2004. Computational identification and characterization of novel genes from legumes. Plant Physiol. 135:1179-1197.

Györgyey, J., Vaubert, D., Jiménez-Zurdo, J. I., Charon, C., Troussard, L., Kondorosi, Á., and Kondorosi, É. 2000. Analysis of Medicago trunca- 
tula nodule expressed sequence tags. Mol. Plant-Microbe Interact. 13:62-71.

Kaijalainen, S., Schroda, M., and Lindstrom, K. 2002. Cloning of nodulespecific cDNA of Galega orientalis. Physiol. Plant. 114:588-593.

Kardailsky, I., Yang, W. C., Zalensky, A., van Kammen, A., and Bisseling, T. 1993. The pea late nodulin gene PsNOD6 is homologous to the early nodulin genes PsENOD3/14 and is expressed after the leghaemoglobin genes. Plant Mol. Biol. 23:1029-1037.

Kato, T., Kawashima, K., Miwa, M., Mimura, Y., Tamaoki, M., Kouchi, H., and Suganuma, N. 2002. Expression of genes encoding late nodulins characterized by a putative signal peptide and conserved cysteine residues is reduced in ineffective pea nodules. Mol. Plant Microbe Interact. 15:129-137.

Kevei, Z., Vinardell, J. M., Kiss, G. B., Kondorosi, A., and Kondorosi, E. 2002. Glycine-rich proteins encoded by a nodule-specific gene family are implicated in different stages of symbiotic nodule development in Medicago spp.. Mol. Plant-Microbe Interact. 15:922-931.

Klotman, M. E., and Chang, T. L. 2006. Defensins in innate antiviral immunity. Nat. Rev. Immunol. 6:447-456.

Kouchi, H., Shimomura, K., Hata, S., Hirota, A., Wu, G. J., Kumagai, H., Tajima, S., Suganuma, N., Suzuki, A., Aoki, T., Hayashi, M., Yokoyama, T., Ohyama, T., Asamizu, E., Kuwata, C., Shibata, D., and Tabata, S. 2004. Large-scale analysis of gene expression profiles during early stages of root nodule formation in a model legume, Lotus japonicus. DNA Res. 11:263-274.

Kumar, S., Tamura, K., and Nei, M. 2004. MEGA3: Integrated software for molecular evolutionary genetics analysis and sequence alignment. Brief. Bioinform. 5:150-163.

Lavin, M., Herendeen, P. S., and Wojciechowski, M. F. 2005. Evolutionary rates analysis of Leguminosae implicates a rapid diversification of lineages during the tertiary. Syst. Biol. 54:530-549.

Maxwell, A. I., Morrison, G. M., and Dorin, J. R. 2003. Rapid sequence divergence in mammalian beta-defensins by adaptive evolution. Mol. Immunol. 40:413-421.

Mergaert, P., Nikovics, K., Kelemen, Z., Maunoury, N., Vaubert, D., Kondorosi, A., and Kondorosi, E. 2003. A novel family in Medicago truncatula consisting of more than 300 nodule-specific genes coding for small, secreted polypeptides with conserved cysteine motifs. Plant Physiol. 132:161-173.

Mergaert. P., Uchiumi, T., Alunni, B., Evanno, G., Cheron, A., Catrice, O., Mausset, A. E., Barloy-Hubler, F., Galibert, F., Kondorosi, A., and Kondorosi, E. 2006. Eukaryotic control on bacterial cell cycle and differentiation in the Rhizobium-legume symbiosis. Proc. Natl. Acad. Sci. U.S.A. 103:5230-5235.

Meyers, B. C., Kozik, A., Griego, A., Kuang, H., and Michelmore, R. W. 2003. Genome-wide analysis of NBS-LRR-encoding genes in Arabidopsis. Plant Cell 15:809-834.

Olivera, B. M. 2006 Conus peptides: Biodiversity-based discovery and exogenomics. J. Biol. Chem. 281:31173-31177.

Ötvös, L., Jr. 2000. Antibacterial peptides isolated from insects. J. Pept. Sci. 6:497-511.
Rodriguez de la Vega, R. C., and Possani, L. D. 2005. Overview of scorpion toxins specific for $\mathrm{Na}^{+}$channels and related peptides: Biodiversity, structure-function relationships and evolution. Toxicon 46:831844.

Sachetto-Martins, G., Franco, L. O., and de Oliveira, D. E. 2000. Plant glycine-rich proteins: A family or just proteins with a common motif? Biochim. Biophys. Acta 1492:1-14.

Scheres, B., van Egelen, F., van der Knaap, E., van de Wiel, C., van Kammen, A., and Bisseling, T. 1990. Sequential induction of nodulin gene expression in developing pea nodule. Plant Cell 2:687-700.

Schopfer, C. R., Nasrallah, M. E., and Nasrallah, J. B. 1999. The male determinant of self-incompatibility in Brassica. Science 286:16971700 .

Schutte, B. C., Mitros, J. P., Bartlett, J. A., Walters, J. D., Jia, H. P., Welsh, M. J., Casavant, T. L., and McCray, P. B., Jr. 2002. Discovery of five conserved beta-defensin gene clusters using a computational search strategy. Proc. Natl. Acad. Sci. U.S.A. 99:2129-2133.

Semple, C. A., Rolfe, M., and Dorin, J. R. 2003. Duplication and selection in the evolution of primate beta-defensin genes. Genome Biol. 4:R31.

Stacey, G., Libault, M., Brechenmacher, L., Wan, J., and May, G. D. 2006. Genetics and functional genomics of legume nodulation. Curr. Opin. Plant Biol. 9:110-121.

Silverstein, K. A., Graham, M. A., Paape, T. D., and VandenBosch, K. A. 2005. Genome organization of more than 300 defensin-like genes in Arabidopsis. Plant Physiol. 138:600-610.

Silverstein, K. A., Graham, M. A., and VandenBosch, K. A. 2006. Novel paralogous gene families with potential function in legume nodules and seeds. Curr. Opin. Plant Biol. 9:142-146.

Watanabe, M., Ito, A., Takada, Y., Ninomiya, C., Kakizaki, T., Takahata, Y., Hatakeyama, K., Hinata, K., Suzuki, G., Takasaki, T. Satta, Y., Shiba, H., Takayama, S., and Isogai, A. 2000. Highly divergent sequences of the pollen self-incompatibility (S) gene in class-I S haplotypes of Brassica campestris (syn. rapa) L. FEBS (Fed. Eur. Biochem. Soc.) Lett. 473:139-144.

Wojciechowski, M. F., Lavin, M., and Sanderson, M. J. 2004. A phylogeny of legumes (Leguminosae) based on analysis of the plastid matK gene resolves many well-supported subclades within the family. Am. J. Bot. 91:1846-1862.

\section{AUTHOR-RECOMMENDED INTERNET RESOURCES}

BioEdit website: www.bioedit.com

CBS prediction server SignalP program: www.cbs.dtu.dk/services

EMBnet Boxshade 3.21 program:

www.ch.embnet.org/software/BOX_form.html

Invitrogen website: www.invitrogen.com

Medicago truncatula website: www.medicago.org

Medicago truncatula Sequencing Resources website: www.medicago.org/genome/cvit_blast.php

NCBI nonredundant database: www.ncbi.nlm.nih.gov/BLAST 